\title{
Inadequate gestational weight gain and malnutrition- related causes of infant death
}

\author{
Regina R Davis-Moss ${ }^{*}$ and Sandra L Hofferth ${ }^{2}$ \\ ${ }^{1}$ Associate Executive Director, American Public Health Association, Washington, DC 20001, USA \\ ${ }^{2}$ Research Professor, Maryland Population Research Center, University of Maryland, College Park, MD 20742, USA
}

\begin{abstract}
Objectives: To examine the association between inadequate gestational weight gain (GWG) and risk of infant death from nutritionally-linked causes of mortality.

Methods: Maternal and infant data from 6,456 women with a singleton, live birth that died in infancy were obtained from the 2005 Birth Cohort Linked BirthInfant Death Data File. The International Classification of Diseases, 10th revision was used to group causes of infant deaths (based on the death certificate) into four categories. GWG was categorized using the 2009 Institute of Medicine (IOM) Pregnancy Weight Gain guidelines. Descriptive and multinomial logistic regression analyses models were fit to estimate odds of infant death associated with nutritionrelated causes compared to infant death from external or unlikely causes.

Results: Only $31 \%$ of women gained the IOM recommended amount of weight during pregnancy. Infants of women with low GWG had adjusted odds of infant mortality from disorders related to length of gestation and fetal malnutrition that were 4.73 times those of deaths from congenital malformations or accidents/injuries. The low GWG-related adjusted odds of deaths in the first year of life from respiratory distress or other respiratory conditions were 2.02 times external or unlikely causes. Inadequate GWG was not associated with the adjusted odds of death from SIDS.

Conclusion: Nutrient deficiency is a biologically plausible mechanism through which inadequate GWG may be associated with an elevated risk of infant death. Improving the quality and quantity of the maternal diet and monitoring weight gain may contribute to a reduction in infant mortality.

Significance: Recent epidemiologic studies have provided evidence linking gestational weight gain (GWG) with risk of infant mortality, but questions remain about the biological pathways. One such question is whether this association is at least partially due to the lasting effects of poor nutrition during pregnancy that are assumed to be related to inadequate GWG. We examined whether inadequate GWG is associated with causes of mortality that can reasonably be considered a consequence of fetal malnutrition. In addition, we examined variability in the link between inadequate GWG and nutrition-related causes of mortality over the infancy period.
\end{abstract}

\section{Introduction}

Weight gain during pregnancy is a public health concern. Recent epidemiologic studies have provided strikingly consistent evidence linking gestational weight gain (GWG) with risk of infant mortality [14]. The initial study in this area of inquiry established an association between inadequate GWG and elevated risk of mortality among infants in the 1988 U.S. National Maternal and Infant Health Survey $(\mathrm{N}=11,558)$ [4]. This association was replicated among a sample of 100,000 randomly selected U.S. infants who were born in 2002 [1].

Recent studies suggest the existence of a moderating effect of maternal pre-pregnancy BMI on the association between GWG and risk of infant mortality [2,3]. Using Pennsylvania linked birth-infant death records (2003-2011; N=1,232,346), [3] Bodnar et al. reported a U-shaped association between inadequate GWG (adjusted for gestational age) and risk of infant mortality. An elevated risk of infant mortality associated with inadequate GWG observed among women with low pre-pregnancy BMI is mitigated among women with average pre-pregnancy BMI, but the risk of infant mortality [associated with low GWG] rises again with increasing pre-pregnancy BMI. Among a sample from phase 5 of the Pregnancy Risk Assessment Monitoring System (2004-2008 N=159,244), Davis et al. found a similar association between low GWG and risk of mortality independent of both gestational age and birthweight [2]. Given that gestational age and birthweight are well-established correlates of GWG [5], it is critical to establish a link between inadequate GWG and infant mortality that is not mediated by poor birth outcomes. Despite the consistent epidemiologic evidence linking low GWG with the risk of infant mortality, questions remain about the biological pathways. As also noted by other observers, a critical question is whether this association is at least partially due to the lasting effects of poor nutrition during pregnancy that are assumed to be related to inadequate GWG [3]. The single study to examine this potential pathway did so by correlating maternal dietary quality during pregnancy and GWG but did not also examine pregnancy outcomes [6]. Lacking a direct test of whether low GWG is associated with infant mortality through nutritional deficiencies in utero, we examine causes of death, defined as the "disease or injury which initiated the train of morbid events leading directly to death" [7]. To test our hypothesis of a link between inadequate GWG and nutrition related infant death, we examine whether inadequate or "low" GWG is associated with causes of mortality that can be reasonably considered to be a consequence of

*Correspondence to: Regina R Davis-Moss, American Public Health Association, 800 I Street, NW, Washington, DC 20001, USA, Tel: (202) 483 1403; E-mail: regina.davis@att.net

Key words: infant mortality, gestational weight gain, ICD-10, cause of death - institute of medicine

Received: June 15, 2018; Accepted: June 21, 2018; Published: June 28, 2018 
fetal malnutrition and not with other causes of mortality. Such evidence of a link between inadequate GWG and specifically, nutrition-related causes of infant death would be in accord with evidence that quality and quantity of nutrition during pregnancy influences fetal development and has long term consequences, perhaps as early as infancy $[4,8,9,10]$. In addition, we examine variability in the link between inadequate GWG and nutrition-related causes of mortality over the infancy period, as deaths during the first month of life are typically associated with conditions surrounding the prenatal period.

\section{Methods}

We analysed data from the 2005 cohort linked file from the National Center for Health Statistics (NCHS). These data include information from birth and death certificates for all infants born in the United States in 2005, including infant date of birth, exact age at death, and International Classification of Diseases, 10th revision (ICD-10) codes for diagnoses reported by the attending physician as related to the underlying cause of death. For the 2005 cohort linked file, $98.7 \%$ of infant death records were linked to their corresponding birth certificates by the NCHS. Additional details about the cohort linked file are published elsewhere [11]. Utilizing data from 10,959 singleton infants who died during the first year of life, we examined the association between low maternal GWG and four categories of infant mortality as defined by ICD-10, coded into UCR130 categories on the NCHS files (NCHS, 2010). Two of these categories include mortality from: 1) "Disorders related to length of gestation and fetal malnutrition" and 2) "Respiratory distress and other respiratory conditions originating in the perinatal period." Deaths identified with UCR codes 86-91 for 'length of gestation and fetal malnutrition' include infants, not light or small for gestational age, showing signs of fetal malnutrition, such as dry, peeling skin and loss of subcutaneous tissue, coded as 087 in the UCR130 classification system on the linked file [12]. This category also includes deaths associated with extremely low birthweight or extreme immaturity (089) and other low birthweight or preterm (090). As noted in the coding manual, these codes are not to be assigned as the main disease or condition unless no other condition is known, and birthweight is to be prioritized over prematurity [12]. Deaths identified with UCR codes 93-104 for "Respiratory distress and other respiratory conditions originating in the perinatal period" include conditions such as congenital pneumonia, chronic respiratory disease, and respiratory distress. We examined these conditions because in utero malnutrition has been found to influence an infant's respiratory system through alveolar loss and enlargement, decrease in diaphragm strength, and infection susceptibility $[13,14]$. In utero reductions in nutrients and oxygen supply can have multiple and lasting negative effects on the developing lung susceptibility [13]. Experimental evidence reveals an association between reduced nutrient availability and stunted lung development (mainly alveolar development and surfactant production) during late gestation leading to impaired gas exchange $[14,15]$. Furthermore, to the extent that normal alveoli septation processes that increase gas exchange surface area occur 36 weeks to term, and continue postnatally, the effect of in utero insult to lung development may be exacerbated after birth [16]. To assess the specificity of association between inadequate GWG and infant mortality we also selected two categories of infant mortality identified by UCR codes that are unlikely to be linked with pathologies that can arise from gestational malnutrition: 1) Sudden infant death syndrome (SIDS, UCR135) and 2) a combined category including unintentional injuries and congenital abnormalities. Although there is no evidence of a link between malnutrition (either during gestation or infancy) and SIDS, maternal malnutrition is associated with risk of low birthweight, which in turn, is linked with risk of SIDS [5]. An examination of the association between inadequate GWG and SIDS net of birthweight allows a test of whether there is a direct association.

"Congenital Malformations" (UCR 118-120, 122-133) and "Accidents and Injuries" (UCR138-156) were combined to create a category of deaths that cannot be reasonably linked with GWG, thus, allowing us to determine specificity of association between inadequate GWG and specific death outcomes. We note that Spina Bifida (UCR121) was excluded from this category because it is linked with inadequate

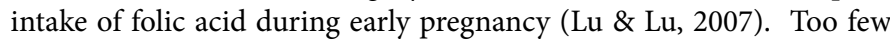
infants in this sample $(\mathrm{N}=12)$ died of spina bifida to permit its analysis as a separate outcome.

The Institute of Medicine has issued gestational weight gain recommendations specific to a woman's pre-pregnancy body mass index of 28-40 lbs. for women who are underweight ( $<18.5 \mathrm{BMI}) ; 25-$ $35 \mathrm{lbs}$. for women of normal weight (18.5-24.9 BMI); 15-25 lbs. for women who are overweight (25-29.9 BMI), and 11-20 lbs. for women who are obese (>=30.0 BMI) (IOM, 2009). We used the number of pounds the mother reported having gained during pregnancy, to classify GWG into three categories based on the lower limit of the 2009 IOM recommended ranges: inadequate $(<24 \mathrm{lbs}$.), normal (between 25 and $40 \mathrm{lbs}$.), or excessive ( $>41 \mathrm{lbs}$.). We focused on the association between inadequate GWG (versus normal or excessive) and cause of infant death because women of low ( $<16$ lbs.) GWG are at higher risk for fetal malnutrition.

Potential confounders include maternal age (35 and older vs. under 35), race/ethnicity (Black, Hispanic vs. White and other), education (high school, some college, college degree vs. less than high school), marital status (married vs. not married), and parity (some versus no previous live births), all of which have been linked to infant mortality. In addition, maternal health conditions such as diabetes (yes/no) and hypertension (yes/no) may be linked to the specific cause of infant death other than through gestational weight gain and are included in the regression models. Finally, we control for gestational age of the infant at birth to adjust the amount of time for adequate weight gain. Consistent with the assumption in the ICD coding guidelines that low birthweight has an intermediate influence on infant death, but is not the underlying cause, we included birthweight as another confounder, measured as weight below 2500 grams.

Using multinomial logistic regression, the four causes of infant mortality were regressed on inadequate GWG, with the infants who died from congenital malformations or accidents/injuries $(\mathrm{N}=2,888)$ serving as the reference group. Models were first fit without covariates. The second model included all the covariates. In addition, as a sensitivity analysis, we restricted our analytic sample to normal weight infants and examined whether low GWG is a statistically significant predictor of mortality among this population. We further reasoned that any association between low GWG and infant mortality due to an underlying experience of poor nutrition in utero, would be more apparent during the early neonatal period than later infancy Separate regressions were fit to estimate the risk of infant death in less than 1 day ( $<24$ hours), during the neonatal period ( 1 to $<28$ days), and during the post-neonatal period ( 28 to $<365$ days) for each cause of death compared with deaths from congenital malformations and accidents/ injuries. Statistical analyses were conducted using SAS Software version 9.2 [16]. Appropriate population weights provided by NCHS were used in all analyses. 


\section{Results}

Descriptive characteristics of the analytic sample are shown in Table 1. Mothers of the 6,456 infants in our analytic sample gained an average of $22 \mathrm{lbs}$. during their pregnancies. Fifty eight percent of women experienced inadequate weight gain, $41.90 \%$ gained at least the recommended amount of weight. Of the infants that died, $38.83 \%$ died during the 24 hours, $24.39 \%$ died between 1 and 28 days, and $36.78 \%$ died between 28 and 365 days. Given the infants' adverse birth outcome, it is not surprising that $58.62 \%$ were born low birthweight and $23.49 \%$ were preterm. Of the sample, $23.49 \%$ died of disorders related to fetal malnutrition and gestation, $14.31 \%$ of respiratory distress, $17.46 \%$ of SIDS and $44.74 \%$ of congenital

Table 1. Descriptive Statistics of Mothers and Infants Who Died, Birth Cohort Linked File, 2005 weighted

\begin{tabular}{|c|c|}
\hline Variable & $\%$ \\
\hline \multicolumn{2}{|l|}{ Mortality } \\
\hline Died $<1$ day & 38.83 \\
\hline Died $1-<28$ days & 24.39 \\
\hline Died $28-<365$ days & 36.78 \\
\hline \multicolumn{2}{|l|}{ Gestational Weight Gain } \\
\hline Inadequate & 58.09 \\
\hline Normal or Excessive & 41.90 \\
\hline \multicolumn{2}{|l|}{ Birth weight, grams } \\
\hline Normal $(>2500 \mathrm{~g})$ & 41.38 \\
\hline Low $(<2500 \mathrm{~g})$ & 58.62 \\
\hline \multicolumn{2}{|l|}{ Gestational Age, weeks } \\
\hline Term Birth (>36 weeks) & 76.51 \\
\hline Preterm Birth & 23.49 \\
\hline \multicolumn{2}{|l|}{ Maternal Age, years } \\
\hline$<34$ & 87.06 \\
\hline$>35$ & 12.94 \\
\hline \multicolumn{2}{|l|}{ Maternal Education, years } \\
\hline Less than High School $(<12)$ & 26.93 \\
\hline Completed High School (=12) & 37.59 \\
\hline Some College (13-15) & 19.30 \\
\hline Completed College (>16) & 16.18 \\
\hline \multicolumn{2}{|l|}{ Maternal Race/Ethnicity } \\
\hline White or Other & 56.68 \\
\hline African American & 30.42 \\
\hline Hispanic & 12.90 \\
\hline \multicolumn{2}{|l|}{ Marital Status } \\
\hline Married & 46.98 \\
\hline Other & 53.02 \\
\hline \multicolumn{2}{|l|}{ Diabetes during Pregnancy } \\
\hline Yes & 3.79 \\
\hline No & 96.21 \\
\hline \multicolumn{2}{|l|}{ Hypertension during Pregnancy } \\
\hline Yes & 4.26 \\
\hline No & 95.74 \\
\hline \multicolumn{2}{|l|}{ Number of Previous Live Births } \\
\hline 0 & 30.87 \\
\hline 1 or more & 69.13 \\
\hline \multicolumn{2}{|l|}{ Cause of Death (based on ICD-10) } \\
\hline Disorders re Fetal Malnutrition and Gestation & 23.49 \\
\hline Respiratory Distress & 14.31 \\
\hline SIDS & 17.46 \\
\hline Congenital malformations, Injuries/Accidents & 44.74 \\
\hline Mean Maternal Weight Gain & $22.45 \mathrm{lbs}$ \\
\hline Mean Maternal Age & 26.14 years \\
\hline Sample Size & 6,456 \\
\hline
\end{tabular}

Malformations, unintentional injuries, or external causes. Mothers had a mean age of 26.14 years; $87 \%$ were age 34 or younger at birth.As presented in Table 2, over the first year of life, infants of women with inadequate

Gestational weight gain had odds of mortality from disorders related to fetal malnutrition and length of gestation that were $9.56(95 \%$ CI: 8.02-11.40) times those of deaths from congenital malformations or accidents. Although the odds were lower, they remained significant after adjustment for medical, behavioral, socioeconomic factors, and low birthweight ( $\mathrm{OR}=4.73,95 \% \mathrm{CI}$ : 3.88-5.77). A similar association held during the first day of life, in the neonatal period, and in the post-neonatal period, even after adjusting for confounding factors. The adjusted odds ratios were larger during the neonatal period, both immediately after birth and within 28 days, compared with the postneonatal period. For example, the adjusted risk of an infant of a low GWG woman dying from fetal malnutrition and gestation-related disorders was 3.96 (95\% CI: 3.05, 5.15) during the first 24 hours, 3.71 (95\% CI: 2.24, 6.16) during the neonatal period, and 2.91 (95\% CI: 1.54$5.50)$ in the post-neonatal period. The odds of deaths in the first year of life from respiratory distress or other respiratory conditions in the crude model were significant ( $\mathrm{OR}=2.81,95 \% \mathrm{CI}: 2.39,3.29)$. After adjustment for covariates, odds were smaller but still significant $(\mathrm{OR}=$ $2.02,95 \%$ CI: $1.70,2.39$ ).

In contrast to disorders related to fetal malnutrition and gestation, the odds of death from respiratory distress were larger for postneonatal deaths compared with deaths in the neonatal period. For example, the adjusted risk of an infant dying from respiratory distress or other related conditions was 2.06 (95\% CI: 1.49, 2.84) during the first day, 1.87 (95\% CI: 1.45, 2.4) during 1-28 days, and 2.83 (95\% CI: 1.844.35) during the post-neonatal period. Infant death from SIDS was associated with inadequate GWG during the first year of life, but not in the direction anticipated. Infants of women with inadequate GWG were $28 \%$ less likely than infants of women with adequate GWG to die of SIDS (OR $=0.72,95 \%$ CI: 0.630 .83 ) than of congenital malformations and accidents during the first day of life. However, once covariates were included in the model, this association was no longer statistically significant $(\mathrm{OR}=0.94,95 \% \mathrm{CI}: 0.80-1.09)$. The same independence between low GWG and risk of deaths from SIDS was observed in the adjusted models for neonatal and post-neonatal periods. Finally, in a sensitivity analysis (data not shown) focusing only on infants born of normal weight $(\mathrm{N}=2,669)$, the adjusted odds of infant mortality from causes related to fetal malnutrition were 6.31 time those of deaths from congenital malformations or accidents (95\% CI: 1.7123.25). However, low GWG was independent of risk of infant mortality due to respiratory causes or SIDS in this sample.

\section{Discussion}

We examined whether inadequate GWG is associated with infant mortality due to two potentially nutrition-related causes: Fetal malnutrition and inadequate development of the respiratory system. In our sample of infants who died at any point during infancy, infants exposed to inadequate GWG were more likely to have their deaths attributed to disorders related to fetal malnutrition and length of gestation or respiratory disorders than to congenital malformations or accidents. These associations held when we restricted the analysis to infants born with normal weight (malnutrition causes only). Inadequate GWG was not more likely to be related to the risk of infant mortality from SIDS, an association for which there was no previous prior evidence, than of infant mortality from congenital malformation 
Table 2. Multinomial logistic regression of cause of death on inadequate gestational weight gain, by timing of death ( $<1$ day, $1-<28$ days, $28-<365$ days, and full infancy period), weighted

\begin{tabular}{|c|c|c|c|c|}
\hline & $\begin{array}{l}\text { Disorders related to } \\
\text { fetal malnutrition and } \\
\text { gestation }(n=1,518)\end{array}$ & $\begin{array}{l}\text { Respiratory distress or other } \\
\text { respiratory condition }(n=925)\end{array}$ & $\begin{array}{c}\text { SIDS } \\
(n=1,125)\end{array}$ & $\begin{array}{l}\text { Congenital malformations } \\
\text { and Injuries/Accidents } \\
(\mathbf{n}=\mathbf{2 , 8 8 8 )}\end{array}$ \\
\hline Day of Death & OR $(95 \% \mathrm{CI})$ & OR $(95 \% \mathrm{CI})$ & OR $(95 \% \mathrm{CI})$ & OR $(95 \% \mathrm{CI})$ \\
\hline \multicolumn{5}{|c|}{ Full Infancy period: Birth to $<365$ days $(n=6,456)$} \\
\hline Low GWG & $9.56(8.02,11.40)^{* * *}$ & $2.81(2.39,3.29)^{* * * *}$ & $0.72(0.63, .83)^{* * *}$ & Reference \\
\hline Low GWG+covariates* & $4.73(3.88,5.77)^{* * * *}$ & $2.02(1.70,2.39)^{* * * *}$ & $0.94(0.80,1.09)$ & Reference \\
\hline \multicolumn{5}{|c|}{ Infancy divided by Length of Time from Birth } \\
\hline \multicolumn{5}{|l|}{ Less than 1 day $(n=2,378)$} \\
\hline Low GWG & $6.29(4.99,7.92)^{* * * *}$ & $2.30(1.69,3.12)^{* * * *}$ & $0.12(0.01,0.97)^{*}$ & Reference \\
\hline Low GWG+covariates* & $3.96(3.05,5.15)^{* * * *}$ & $2.06(1.49,2.84)^{* * * *}$ & $0.17(0.02,1.55)$ & Reference \\
\hline \multicolumn{5}{|c|}{ Neonatal period: 1 to $<28$ days $(n=1,575)$} \\
\hline Low GWG & $7.98(4.99,12.75)^{* * *}$ & $2.53(2.01,3.18)^{* * * *}$ & $0.74(0.48,1.16)$ & Reference \\
\hline Low GWG+covariates* & $3.71(2.24,6.16)^{* * * *}$ & $1.87(1.45,2.43)^{* * * *}$ & $1.01(0.63,1.62)$ & Reference \\
\hline \multicolumn{5}{|c|}{ Post-neonatal period - 28 to $<365$ days $(n=2,503)$} \\
\hline Low GWG & $4.46(2.45,8.08)^{* * * *}$ & $3.78(2.54,5.62)^{* * * *}$ & $0.96(0.81,1.13)$ & Reference \\
\hline Low GWG+covariates* & $2.91(1.54,5.50)^{* * *}$ & $2.83(1.84,4.35)^{* * * *}$ & $1.04(0.87,1.24)$ & Reference \\
\hline
\end{tabular}

"Covariates include gestational age, maternal age, race/ethnicity, education, marital status, diabetes, hypertension, birth weight, and parity.

+Infants who died from congenital birth defects (omitting spina bifida) or accidents/injuries served as the reference group.

\pm Underlying causes of death were assigned to one of the following UCR130 categories of death used by NCHS: 1: 086-091, 2: 093-104, 3:

135, 4: 118-133 (except 121) and 138-156.

${ }_{* * *} \mathrm{p}<.001 ; * * \mathrm{p}<.01 ; * \mathrm{p}<.05$

or accidents. The association between inadequate GWG and elevated risk of death due to disorders related to fetal malnutrition was strongest in the neonatal period and remained, though attenuated, during the post-neonatal period. A similar gradient was not observed for mortality due to respiratory causes. The strongest association of GWG with mortality due to respiratory distress was observed in the postneonatal period. We suspect that the availability of new interventions for infants with respiratory problems such as surfactant therapy during the early neonatal period may have reduced, but not eliminated, risk of death during the immediate post-partum period [18]. These therapies may suppress but not completely eliminate risk later in infancy.

The present study contributes to the scant literature on the association between GWG and infant mortality by using nationally representative data to examine the association between maternal weight gain and infant mortality. However, several limitations of the study must be noted. First, in 2005 the majority of the states were still using the 1989 standard birth certificate which lacked data on maternal pre-pregnancy weight and height. Thus, overweight or obese women who achieved the recommended weight gain could have been incorrectly classified as inadequate in this research. There is also the possibility that a woman's gestational weight gain could be misreported or misrecorded. However, this misclassification error is likely to occur at random as it is not linked with the probability of mortality. Second, although we controlled for several known confounders, dietary habits could not be addressed. Information on nutrition intake would ideally have been included. Third, evidence suggests that the rate of gestational weight gain during pregnancy may be as or more important to adverse birth outcomes than the total amount of gestational weight gain [19]. The available information did not lend itself to assessing the rate of gain during pregnancy or the risk of infant mortality among women with inadequate weight gain by trimester. Gestational age at birth was included as an adjustment for this problem. Fourth, the classification by broad causes of death may have also decreased the precision of estimation. In particular, physician classification of cause of death as fetal malnutrition is often indirect, based upon low birthweight and prematurity rather than on actual verification of abnormal fetal growth or malnutrition [20].
These methodological limitations are not unique to the present study, but rather reflect the limitations of existing data sources. The nationwide adoption of the 2003 revised U.S. Certificate of Live Birth may yield more useful information for maternal weight gain research in the very near future. The Birth Cohort Linked Birth-Infant Death Data File will continue to be the definitive source of information on U.S. births and infant deaths for federal agencies and researchers. Our findings provide tentative support for the notion that inadequate GWG may be associated with greater risk of infant mortality beyond that associated with low birthweight. Should our results be replicated in subsequent studies, it would support further research as to the manner and extent that inadequate nutrient supply in utero can influence later health $[21,22]$.

The results of this study have meaningful public health and clinical implications. Maternal weight gain is susceptible to intervention. Improving the quality and quantity of the maternal diet as well as the advice women receive about GWG, and monitoring their weight and nutritional intake, may contribute to a reduction in infant mortality.

\section{References}

1. Davis RR, Hofferth SL (2012). The association between inadequate gestational weight gain and infant mortality among U.S. infants born in 2002. Matern Child Health J, 16:119-124. [Crossref]

2. Davis R, Hofferth S, Shenassa E (2014). Gestational weight gain and risk of infant mortality in the United States. Am J Public Health, 104:S90-S95.

3. Bodnar LM, Siminerio LL, Himes KP, et al. (2016) Maternal obesity and gestational weight gain are risk factors for infant death. Obesity (Silver Spring) 24: 490-498. [Crossref]

4. Chen A, Feresu SA, Fernandez C, Rogan WJ (2009) Maternal obesity and the risk of infant death in the United States. Epidemiology 20: 74-81. [Crossref]

5. $\mathrm{Lu} \mathrm{M}, \mathrm{Lu}, \mathrm{J}$ (2007). Maternal Nutrition and Infant Mortality in the Context of Relationality. The Courage to Love: Infant Mortality Commission. Implications for Care, Research, and Public Policy to Reduce Infant Mortality Rates (pp. 1-76). Washington, DC: Joint Center for Political and Economic Studies Health Policy Institute.

6. Shin D, Bianchi L, Chung H, Weatherspoon L, Song W (2014). Is gestational weight gain associated with diet quality during pregnancy? Matern Child Health $J$, 18: 14331443 
7. World Health Organization. (2011). International statistical classification of diseases and related health problems. 10th revision. Volume 2: Instruction manual, 2010 edition (p. 31). Geneva: World Health Organization

8. Schulz L (2010). The Dutch Hunger Winter and the developmental origins of health and disease. Proc Natl Acad Sci U S A, 107:16881-16886. [Crossref]

9. Institute of Medicine and National Research Council. (2009). Weight gain during pregnancy: Reexamining the guidelines. The National Academies Press

10. Abu-Saad K, Fraser D (2010) Maternal nutrition and birth outcomes. Epidemiol Rev 32: 5-25. [Crossref]

11. National Center for Health Statistics. (2005). National Center for Health Statistics linked birth/infant death data set, 2005 cohort data. Hyattsville, Maryland: U.S. Department of Health and Human Services, Centers for Disease Control and Prevention, National Center for Health Statistics.

12. World Health Organization. (2014). International statistical classification of diseases and related health problems. Geneva: WHO Press, World Health Organization

13. Stocks J, Hislop A, Sonnappa S (2013). Early lung development: lifelong effect on respiratory health and disease. Lancet Respir Med., 1:728-742. [Crossref]

14. Kallapur S, Ikegami M. (2006). Physiological consequences of intrauterine insults. Paediatr Respir Rev., 7:110-116. [Crossref]
15. Maritz GS, Morley CJ, Harding R (2005). Early developmental origins of impaired lung structure and function. Early Hum Dev., 81:763-771. [Crossref]

16. Copland I, Post M (2004) Lung development and fetal lung growth. Paediatr Respir Rev 5 Suppl A: S259-264. [Crossref]

17. Kebaish KM, Neubauer PR, Voros GD, Khoshnevisan MA, Skolasky RL (2011) Scoliosis in adults aged forty years and older: prevalence and relationship to age, race, and gender. Spine (Phila Pa 1976) 36: 731-736. [Crossref]

18. McEvoy C, Aschner J (2015). The natural history of bronchopulmonary dysplasia: The case for primary prevention. Clinics in Perinatology, 42: 911-931. [Crossref]

19. Langford A, Joshu C, Chang JJ, Myles T, Leet T (2011). Does gestational weight gain affect the risk of adverse maternal and infant outcomes in overweight women? Matern Child Health J., 15: 860-865. [Crossref]

20. Zhang J, Merialdi M, Platt LD, Kramer MS (2010). Defining normal and abnormal feta growth: promises and challenges. American Journal of Obstetrics and Gynecology, 202: 522-528. [Crossref]

21. Barker DJ (1997) Maternal nutrition, fetal nutrition, and disease in later life. Nutrition 13: 807-813. [Crossref]

22. Gluckman PD, Hanson MA, Cooper C, Thornburg KL (2008) Effect of in utero and early-life conditions on adult health and disease. N Engl J Med 359: 61-73. [Crossref]

Copyright: $@ 2018$ Davis-Moss RR. This is an open-access article distributed under the terms of the Creative Commons Attribution License, which permits unrestricted use, distribution, and reproduction in any medium, provided the original author and source are credited. 